PROCEEDINGS OF THE

AMERICAN MATHEMATICAL SOCIETY

Volume 27, Number 1, January 1971

\title{
EXPLICIT CHARACTERIZATION OF SPHERICAL CURVES
}

\section{SHLOMO BREUER AND DAVID GOTTLIEB}

Abstract. It is shown that the differential equation characterizing a spherical curve can be solved explicitly to express the radius of curvature of the curve in terms of its torsion.

In a recent paper [1], the authors have deduced necessary and sufficient conditions on the (sufficiently smooth) coefficients of the general linear, homogeneous, $n$th order, ordinary differential equation, so that it can be carried into an equation with constant coefficients by a suitable transformation of the independent variable. The transformation is determined and the solutions are given explicitly in terms of the original coefficients whenever the conditions are met.

For our purpose here, we quote a special case of Theorem 4.1 in [1]:

Let the real functions $p(x)$ and $q(x)$ be continuously differentiable and positive throughout some interval $J$. Then a necessary and sufficient condition that the equation

$$
\left[p(x) y^{\prime}\right]^{\prime}+q(x) y=0
$$

can be transformed into an equation with constant coefficients in $J$, through a transformation of the form

$$
\xi=\int a(x) d x
$$

with $a(x)$ continuously differentiable, is that

$$
(d / d x)[p(x) q(x)]^{1 / 2}=c q(x),
$$

where $c$ is an arbitrary real constant.

At most three distinct cases can arise, depending upon the value of $c$ in (3), and they are all treated explicitly in [1]. In particular, if $c=0$, we find from (3) that

$$
p(x) q(x)=D^{-2}
$$

Received by the editors January 30, 1970.

AMS 1969 subject classifications. Primary 5301, 3402.

Key words and phrases. Radius of curvature, torsion, differential equation of spherical curves, explicit solutions of differential equations.

Copyright @ 1971, American Mathematical Society 
where $D^{-2}$ is a positive constant. In that case, Corollary 4.1 in [1] shows that the general solution of (1) is given by

$$
y(x)=A_{1} \cos \left[D \int q(x) d x\right]+A_{2} \sin \left[D \int q(x) d x\right],
$$

where $A_{1}$ and $A_{2}$ are arbitrary constants. This result can of course be verified directly.

We wish to apply the last result to the explicit characterization of spherical curves. Let $s, \rho(s)$ and $\tau(s)$ denote, respectively, the length of arc, the radius of curvature and the torsion of a curve. Then it is well known $[2$, p. 62] that, for $\tau \neq 0$, a necessary and sufficient condition that the curve be a spherical curve is that $\rho(s)$ and $\tau(s)$ satisfy

$$
\frac{d}{d s}\left[\frac{1}{\tau(s)} \frac{d \rho}{d s}\right]+\tau(s) \rho(s)=0 .
$$

The authors are not aware that any explicit relation between $\rho(s)$ and $\tau(s)$ has ever been deduced from (6). However, comparing (6) with (1) and identifying $\rho$ with $y$, we find that $p=1 / \tau, q=\tau$, and consequently from (4) that $D^{-2}=1$. We conclude, therefore, with the aid of (5) that a curve is a spherical curve if and only if $\rho(s)$ and $\tau(s)$ satisfy the explicit relation

$$
\rho(s)=A_{1} \cos \left[\int \tau(s) d s\right]+A_{2} \sin \left[\int \tau(s) d s\right],
$$

$A_{1}$ and $A_{2}$ being arbitrary constants.

\section{RERERENCES}

1. S. Breuer and D. Gottlieb, The reduction of linear ordinary differential equations to equations with constant coefficients, J. Math. Anal. Appl. 31 (1970).

2. E. Kreyszig, Differential geometry, Mathematical Expositions, no. 11, Univ. of Toronto Press, Toronto, 1959. MR 21 \#7507.

Tel Aviv University, Tel Aviv, IsRael 